\title{
Numerical Study of the Momentumless Wake of an Axisymmetric Body
}

\author{
Meng-Huang Lu* and Ana I. Sirviente ${ }^{\dagger}$ \\ University of Michigan, Ann Arbor, MI, 48109-2145
}

\begin{abstract}
A second order turbulence closure is used to calculate the turbulent flow in a swirling axisymmetric momentumless wake. The solutions are compared with recent experimental and numerical data. Simulations corresponding to the component flows (i.e. bare body wake and jet) are also performed with the same turbulence closure. The comparisons with other experimental and numerical data reveal that the predictions of the model for the axisymmetric jet are quite good although larger discrepancies exist for the Reynolds stress tensor for both axisymmetric wake and zero-momentum wake.
\end{abstract}

\section{Nomenclature}

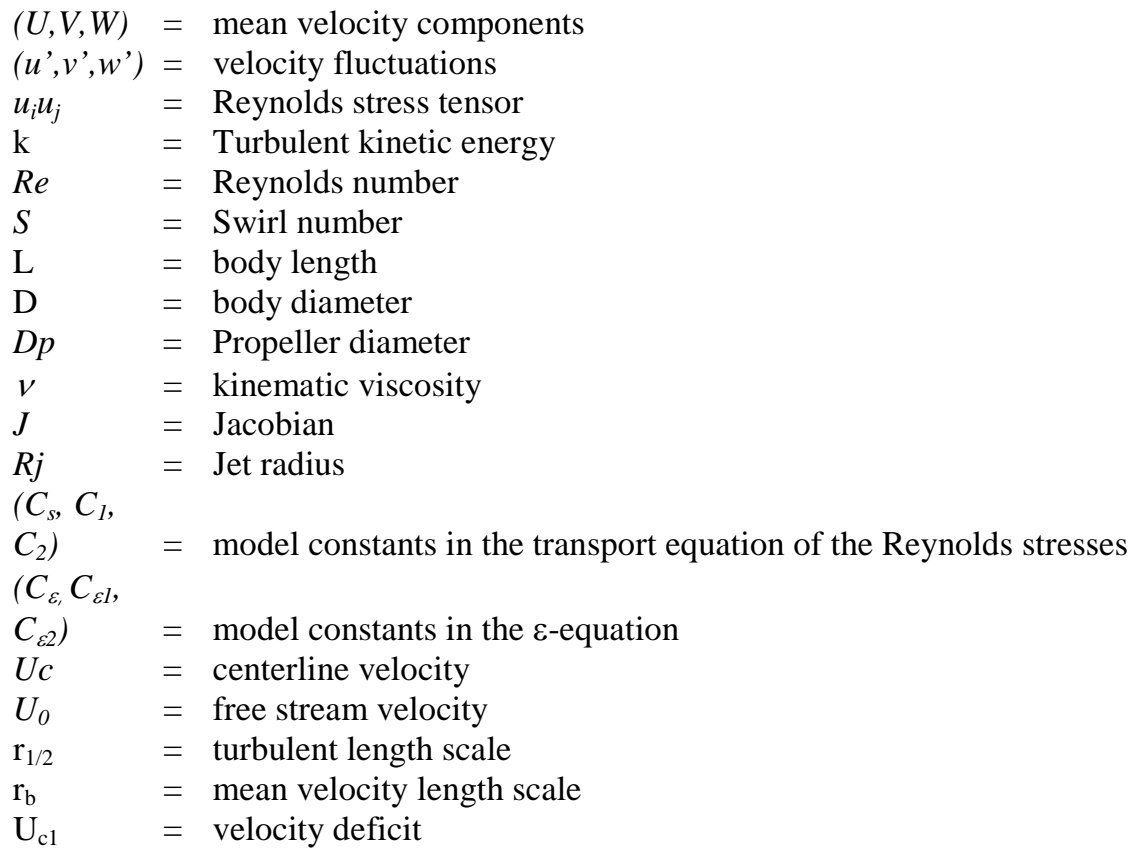

\section{Introduction}

$\mathrm{T}$ He wake of a body is characterized by a momentum deficit and many past studies have characterized the development and decay of the mean and turbulence velocity and length scales representative of such flows. The wake of a three-dimensional body driven by a propeller or a jet, with appendages and controls in operation, is easily among the most complex of shear flows. There are very few detailed analytical or numerical studies, or experimental measurements, in the swirling wake of self-propelled bodies because they present a challenge to modeling as well as instrumentation.

\footnotetext{
* Graduate Research Assistant, Naval Architecture and Marine Engineering Department.

${ }^{\dagger}$ Assistant Professor, Naval Architecture and Marine Engineering Department, asirv@engin.umich.edu, AIAA Senior Member.
} 
Wakes with zero momentum excess are characteristic of self-propelled movement, where the body drag is cancelled by the thrust provided by the means of propulsion, generally a jet or a propeller. The study of such wakes is hindered by the limited experimental data available. This is especially the case, as mentioned by a number of experimental and theoretical studies, when noting that these flows are highly influenced by various conditions of the experiments, such as turbulence level at the inlet or possible small deviations from zero momentum. The plane momentumless wake (i.e. Cimbala and Park ${ }^{1}$, Park and Cimbala ${ }^{2}$, etc.) has however received more attention in the past than the axisymmetric momentumless wake. The studies of Ridjanovic ${ }^{3}$, Wang $^{4}$, Naudascher ${ }^{5}$, in the wake of a circular disk with a jet at its center concluded that the far wake resembles decaying homogeneous turbulence. The study of Higuchi and Kubota ${ }^{6}$ was concerned with the wake of a thin circular tube interacting with a jet adjusted to produce a zero net axial momentum. The studies of Schetz and Jakubowski ${ }^{7}$, Ferry and Piquet ${ }^{8}$, Faure ${ }^{9}$, Hyun and Patel $^{10}$ and Sirviente and Patel ${ }^{11,12}$ are among the few experimental studies on axisymmetric zero momentum wakes of streamlined axisymmetric bodies self-propelled with jets or propellers. Other studies (such as the one by Kostomakha and Lesnova ${ }^{13}$ ) were conducted to experimentally measure the turbulent swirling wake behind a sphere with complete and partial drag compensation. A few experimental studies concerning momentumless wakes in stratified flows exist in the literature, such as Schooley and Stewart ${ }^{14}$, Gilreath and Brandt ${ }^{15}$ and Chernykh et al. ${ }^{16}$ among others.

Similarity studies have been conducted for axisymmetric momentumless wakes in the past ${ }^{17-21}$. The results from them seem to depend strongly on the turbulence model employed in the analysis. A summary of the results of the various studies can be found in Sirviente and Patel ${ }^{11}$. Few attempts at numerically modeling this type of flows can be found in the literature such as the studies of Chernykh et al. ${ }^{22}$ of a swirling momentumless turbulent wake, Chernykh and Voropayeva ${ }^{23}$ where the evolution of a zero momentum wake in a linearly stratified fluid was carried out by using a hierarchy of the second order mathematical model. It was concluded that a satisfactory agreement with experimental data can be obtained when differential transport equations for normal and one of the tangential Reynolds stresses is used along with non-equilibrium algebraic relations for other tangential stresses.

This paper reports on the numerical simulation of the wake of a body of revolution at zero incidence, with a propeller in operation. It documents the development of the momentumless wake corresponding to the experimental study of Hyun and Patel ${ }^{10}$. The numerical method employed solves the Reynolds-Averaged Navier-Stokes (RANS) equations for an incompressible fluid in generalized curvilinear coordinates and a second order turbulence closure is used. The performance of the turbulence model is evaluated in this study by first comparing its results to those reported by Chernykh et al. for a swirling momentumless wake computed with a second order closure that combined differential transport equations with algebraic expressions for the normal and Reynolds stresses. Their comparisons to experimental data indicated very good agreement. Secondly comparisons of the numerical simulation corresponding to the database of Hyun and Patel are performed and the results compared with some numerical simulations of the same flow performed by Stern et al. ${ }^{24}$ using a $\kappa-\varepsilon$ model. The self-propelled wake can be viewed in a very simplistic manner as the combination of two distinct shear layers (i.e. boundary layer and propeller or jet flow). The performance of the second order closure is assessed by numerically simulating the wake corresponding to the same body used in the swirling axisymmetric zero momentum wake of Hyun and Patel but without the propeller. The experimental data corresponding to the bare body condition is used for comparisons. Finally simulations of a fully submerged circular jet are also conduced and the results compared to past experimental data. The purpose of this study is two fold. First to numerically investigate the mometnumless axisymmetric wake of a propeller-driven body with a second order closure. Secondly this study aims at assessing the results obtained from the simulations of various canonical flows (i.e. axisymmetric momentumless wake, axisymmetric wake and jet) by using the same second order turbulent closure in all cases.

\section{Numerical Methods}

The method used solves the Reynolds-averaged Navier-Stokes (RANS) equations in generalized curvilinear coordinates. The velocity components are however expressed in Cartesian coordinates, following a partial transformation approach. The governing equations are discretized in space on a non-staggered grid using second order approximations. The convective terms in the momentum equations are discretized by using second-order accurate upwind differencing, while the pressure gradient and viscous terms in the momentum equations and the divergence operator in the continuity equation make use of second order accurate central finite differencing. A discrete pressure-Poisson equation is used to enforce the incompressibility constraint. The formulation of Sotiropoulos and Abdallah ${ }^{25}$, designed to avoid the odd-even decoupling implicit on non-staggered grids, was used.

The discrete momentum equations are integrated in time to steady state by using a four-stage explicit RungeKutta scheme. The pressure-velocity equation is solved using the alternate-direction-implicit (ADI) approximate 
factorization method to accelerate its convergence, and the Thomas algorithm. Consequently the pressure-Poisson equation is transformed into a diffusion-like evolution equation. Convergence acceleration techniques, such as local time stepping and implicit residual smoothing, are used to enhance the error damping properties of the time marching procedure. This numerical methodology has been tested for a wide range of laminar flows and turbulent flows with various turbulence closures ${ }^{26-28}$. The comparisons of the computed solutions with other numerical results and experimental data demonstrated its ability to simulate those flows with great accuracy.

In this study a second order turbulence closure is used. The Cartesian components of the Reynolds stress tensor, $\overline{u_{i} u_{j}}$, are solved at the same nodes where the velocity vector is solved at. The transport equations for the Reynolds stresses and the $\varepsilon$-equation can be written in generalized curvilinear coordinates, $\xi^{\mathrm{k}}$ where $\mathrm{J}$ is the Jacobian of the geometric transformation, as follows:

$$
\begin{aligned}
& \frac{\partial \overline{\mathrm{u}_{\mathrm{i}} \mathrm{u}_{\mathrm{j}}}}{\partial \mathrm{t}}+\mathrm{V}^{\mathrm{k}} \frac{\partial \overline{\mathrm{u}_{\mathrm{i}} \mathrm{u}_{\mathrm{j}}}}{\partial \xi^{\mathrm{k}}}=-\left[\overline{\mathrm{u}_{\mathrm{i}} \mathrm{u}_{\mathrm{k}}} \frac{\partial \mathrm{U}_{\mathrm{j}}}{\partial \xi^{\ell}} \xi_{\mathrm{x}_{\mathrm{k}}}^{\ell}+\overline{\mathrm{u}_{\mathrm{j}} \mathrm{u}_{\mathrm{k}}} \frac{\partial \mathrm{U}_{\mathrm{i}}}{\partial \xi^{\ell}} \xi_{\mathrm{x}_{\mathrm{k}}}^{\ell}\right]+\left[v \mathrm{~J} \frac{\partial}{\partial \xi^{\mathrm{k}}}\left(\frac{\mathrm{g} \mathrm{k} \ell}{\mathrm{J}} \frac{\partial \overline{\mathrm{u}_{\mathrm{i}} \mathrm{u}_{\mathrm{j}}}}{\partial \xi^{\ell}}\right)\right]+ \\
& +\left[\mathrm{J} \frac{\partial}{\partial \xi^{\mathrm{k}}}\left(\mathrm{C}_{\mathrm{s}} \frac{\mathrm{k}}{\varepsilon} \frac{\xi_{\mathrm{x}_{\ell}}^{\mathrm{m}} \xi_{\mathrm{x}_{\mathrm{n}}}^{\mathrm{k}}}{\mathrm{J}}\left(\overline{\mathrm{u}_{\mathrm{i}} \mathrm{u}_{\ell}} \frac{\partial \overline{\mathrm{u}_{\mathrm{j}} \mathrm{u}_{\mathrm{n}}}}{\partial \xi^{\mathrm{m}}}+\overline{\mathrm{u}_{\mathrm{j}} \mathrm{u}_{\ell}} \frac{\partial \overline{\mathrm{u}_{\mathrm{n}} \mathrm{u}_{\mathrm{i}}}}{\partial \xi^{\mathrm{m}}}+\overline{\mathrm{u}_{\mathrm{n}} \mathrm{u}_{\ell}} \frac{\partial \overline{\mathrm{u}_{\mathrm{i}} \mathrm{u}_{\mathrm{j}}}}{\partial \xi^{\mathrm{m}}}\right)\right)\right] \\
& -\left[\frac{2}{3} \delta_{\mathrm{ij}} \varepsilon\right]+\left[-\mathrm{C}_{1} \varepsilon\left(\frac{\overline{\mathrm{u}_{\mathrm{i}} \mathrm{u}_{\mathrm{j}}}}{\mathrm{k}}-\frac{2}{3} \delta_{\mathrm{ij}}\right)-\mathrm{C}_{2}\left(\mathrm{P}_{\mathrm{ij}}-\frac{2}{3} \delta_{\mathrm{ij}} \mathrm{P}\right)\right] \\
& \frac{\partial \varepsilon}{\partial \mathrm{t}}+\mathrm{V}^{\mathrm{k}} \frac{\partial \varepsilon}{\partial \xi^{\mathrm{k}}}=\left[\mathrm{J} \frac{\partial}{\partial \xi^{\mathrm{k}}}\left(\frac{\xi_{\mathrm{x}_{\mathrm{n}}^{\mathrm{k}}}^{\mathrm{k}} \xi_{\mathrm{x}_{\ell}}^{\mathrm{m}}}{\mathrm{J}}\left(\mathrm{C}_{\varepsilon} \frac{\mathrm{k}}{\varepsilon} \overline{\mathrm{u}_{\mathrm{n}} \mathrm{u}_{\ell}}+v \delta_{\mathrm{n} \ell}\right) \frac{\partial \varepsilon}{\partial \xi^{\mathrm{m}}}\right)\right]+\left[\mathrm{C}_{\varepsilon 1} \frac{\varepsilon}{\mathrm{k}} \mathrm{P}\right]-\left[-\mathrm{C}_{\varepsilon 2} \frac{\varepsilon}{\mathrm{k}} \varepsilon\right]
\end{aligned}
$$

where $\mathrm{V}^{\mathrm{k}}$ are the contravariant components of the mean velocity, while $\mathrm{U}_{\mathrm{k}}$ are the mean Cartesian velocity components and $v$ is the kinematic viscosity. The first and second terms in the right hand side of equation (1) represent the production by mean shear $\mathrm{P}_{\mathrm{ij}}$ and the viscous diffusion respectively and can be computed exactly without modeling. The third term in the right hand side of (1) represents the Reynolds stress turbulent diffusion and is modeled using Hanjalic and Launder ${ }^{29}$ approach. The last term in the right hand side of equation (1) represents the pressure-strain correlation term and is modeled according to the Launder and Shima ${ }^{30}$ proposal. In equation (1) $\mathrm{C}_{\mathrm{s}}$ is a model constant, $\mathrm{C}_{1}$ and $\mathrm{C}_{2}$ are functions of the second and third anisotropy tensor invariants, $\delta_{\mathrm{ij}}$ is Kronecher's delta, $\mathrm{P}$ is the production of turbulence kinetic energy, $\mathrm{k}, \varepsilon$ is the dissipation rate of turbulence energy and the local isotropy assumption is followed to compute the rate of dissipation tensor. $\mathrm{C}_{\varepsilon}, \mathrm{C}_{\varepsilon 1}$ and $\mathrm{C}_{\varepsilon 2}$ are model constants in equation (2).

The latter equations (1) and (2) are discretized in space and integrated in time following a similar procedure to that indicated earlier for the momentum equations. That is a second-order upwind finite differencing scheme is used for the convective terms and second-order accurate central finite differences are used for the rest of the terms. A four-stage Runge-Kutta method is also used to advance the discrete equations in time. In this study the model constants of equations (1) and (2) were $\mathrm{C}_{1}=1.8, \mathrm{C}_{2}=0.6, \mathrm{Cs}=0.22, \mathrm{C}_{\varepsilon}=0.18, \mathrm{C}_{\varepsilon 1}=1.45$, and $\mathrm{C}_{\varepsilon 2}=1.90$.

\section{A. Computational Grid and Boundary Conditions}

In this study the computational domain corresponding to the axisymmetric bare body wake and the axisymmetric momentumless wakes extends 9.38 body lengths downstream and 1.34 body lenghts in the transverse direction. The numerical grid consists of $55 \times 80 \times 8$ grid nodes in the axial, radial and circumferential directions respectively. The grid lines are stretched from the inlet downstream and from the centerline toward the outer boundary by using a hyperbolic tangent stretching function. The computational domain corresponding to the circular jet extends 150 jet diameters downstream and 100 jet diameters in the transverse direction. The numerical grid also consists of $60 \times 70 \times 8$ grid nodes in the axial, radial and circumferential directions respectively. A similar stretching to that mentioned earlier for the wake grids was also used. A grid independence study was performed. The cross-section view of the grid is shown in Figure 1. 


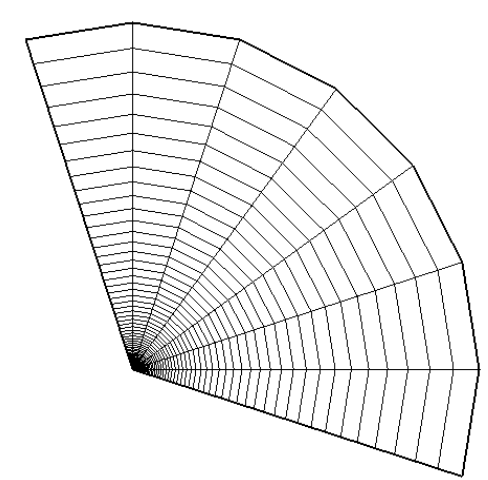

Figure 1. Cross-section view of the computational grid

The boundary conditions were specified as follows. The inlet boundary condition for the axisymmetric wake and momentumless wake was located at $\mathrm{x} / \mathrm{L}=1.00$ and $\mathrm{x} / \mathrm{L}=1.02$ respectively, and the data taken from the experimental results of Hyun and $\mathrm{Patel}^{10}$. Inlet conditions are specified at $\mathrm{x} / \mathrm{D}=3$ for the circular jet and the data from the experimental study of Sami et al. ${ }^{31}$ used. On the symmetry boundaries mirror-image reflection for the grid and the flow variables is used in all cases. The exit boundary condition is imposed in all cases by assuming zero streamwise diffusion and the outer boundary conditions assume free-stream recovery. The simulations presented in this study correspond to Reynolds numbers based on the body length and the free stream velocity of $\operatorname{Re}=1.55 \times 10^{6}$ for the axisymmetric wakes. The Reynolds number based on the jet diameter and the centerline velocity at the exit is $\mathrm{Re}=2.2 \times 10^{5}$ for the circular jet. The CFL number used in these computations is 1.2 and 1.5 for the Reynolds stress equations. In all cases the residuals, defined by the summation of differences between the current and the previous iterations, were reduced by at least four orders of magnitude.

\section{Results and Discussion}

This section is subdivided in three main parts. The first part corresponds to the simulations of an axisymmetric swirling momentumless wake and the corresponding comparisons with past simulations and experimental data. In order to assess the performance of the Reynolds stress closure used in this study, the last two parts of this section present simulations corresponding to an axisymmetric wake and a jet. Those flows can be viewed as component flows of the corresponding momentumless wake and hence the ability of the second order to predict the development of each of them seems critical.

\section{A. Swirling Momentumless Wake}

A self-propelled body is characterized by a balance between the thrust generated by the propulsion system (e.g. propeller, jet, etc.) and the drag generated by the body. The experimental results ${ }^{13}$ corresponding to the axisymmetric zero-momentum turbulent wake behind a sphere are used first. A swirling jet issuing at the back of the sphere compensates the sphere's drag. These experiments were carried out in a low-turbulence closed wind tunnel. The test section was $4 \mathrm{~m}$ long and $0.4 \mathrm{~m} \times 0.4 \mathrm{~m}$ in cross section where a $25 \mathrm{~mm}$ sphere with a special $6 \mathrm{~mm}$ diameter nozzle built into the sphere to form a swirling jet was placed. The measurements were taken with single and crossed hot wire probes to measure the three velocity components as well as the six components of the Reynolds stress tensor. The experiments were carried out at a Reynolds number, Re, based on the sphere diameter and the free-stream velocity of $2.6 \times 10^{4}$ and various swirl numbers, $\mathrm{S}$, defined as the ratio of tangential to axial momentum at the exit:

$$
\mathrm{S}=\frac{\mathrm{M}_{\theta \mathrm{x}}}{\mathrm{M}_{\mathrm{x}}\left(\mathrm{R}_{\mathrm{j}} / \mathrm{D}\right)}
$$


where $\mathrm{M}_{\theta \mathrm{x}}$ is the axial flux of tangential momentum, $\mathrm{M}_{\mathrm{x}}$ is the axial flux of axial momentum, $\mathrm{D}$ is the body's diameter and $R_{j}$ is the nozzle or jet radius. The case chosen for this simulation corresponds to a Swirl number of 2.2 .

Chernykh et al. ${ }^{22}$ performed numerical simulations to validate the experimental data of Kostomakha and Lesnova $^{13}$. The numerical model they used was a RANS solver with various (five different models) second order turbulence closures. The most complete model (model 1, as it was referred to by the authors) was based on the Reynolds stress closure of Launder and Morse ${ }^{32}$. Model 2 corresponds to a simplified version of model 1. Model 3 is based on the use of the algebraic representations of the tangential Reynolds stresses. Model 4 makes use of the algebraic representations of the normal and one of the tangential stresses and model 5 based on the differential transport equations for the normal stresses and the corresponding algebraic expressions for the tangential stresses. The best agreement with the experimental data was found from the use of Model 5. Some of those numerical results are used for comparison with the numerical simulations performed in this study to validate the experimental data of Kostomakha and Lesnova (1995), where the experimental data corresponding to $\mathrm{x} / \mathrm{D}=20$ was used as inlet profile. In all cases the sphere diameter and the free stream velocity are used as corresponding mean axial length and velocity scales.

The results corresponding to the velocity defect (i.e. maximum difference between the free-stream velocity and the axial mean velocity) and the tangential velocity components are shown in Figure 2 . In that figure the experimental data of Kostomakha and Lesnova (1995) at $\mathrm{x} / \mathrm{D}=50$ and the numerical results from Chernykh et al. (1998) corresponding to their best turbulence model for $\mathrm{x} / \mathrm{D}=50$ and 100 are compared with the present simulations. It can be observed that the prediction of the velocity in the outer region of the shear layer is considerably better than that in the inner region. The centerline velocity is greatly over predicted by the present method. In contrast the turbulence approach employed by Chernykh et al. does a much better job all across the flow, but more specifically in the neighborhood of the centerline.

The comparisons corresponding to the turbulent fluctuation intensities of the velocity components are presented in Figure 3. The distributions of the tangential stresses uv and vw are shown in Figure 4. The prediction of the streamwise and radial normal stresses follows a similar trend to that seen for the velocity decay in the outer region of the flow where the recovery of freestream conditions takes place. That is the outer region is better predicted than the central portion of the flow largely dominated by strong velocity gradients. The experimental results as well as the numerical simulations of Chernykh et al. indicate that by $\mathrm{x} / \mathrm{D}=50$ the turbulence is quite isotropic with similar levels of turbulent fluctuation intensities for all the velocity components. The prediction from the turbulence closure employed in this study however shows larger values for the radial velocity fluctuations than those corresponding to the streamwise and tangential velocity fluctuations respectively, showing an important degree of anisotropy. The tangential stresses shown in Figure 4 display a fairly good agreement with the experimental data for vw while the differences are important for uv. Those differences are considerably more significant for the present model than that employed by Chernykh et al.

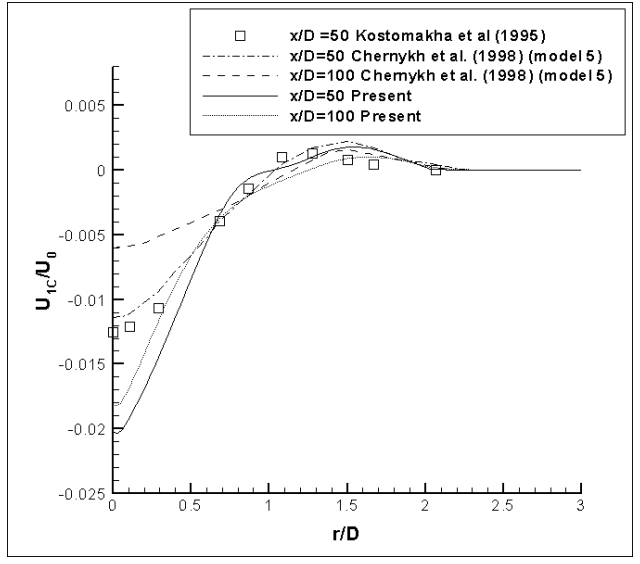

a)

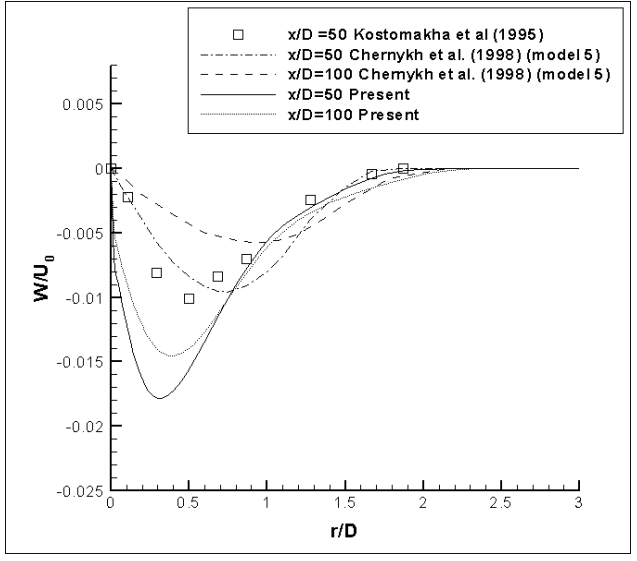

b)

Figure 2. Comparison of a) the calculated dimensionless profiles of the mean velocity defect and b) the tangential velocity with the experimental data of Kostomakha et al. (1995) and the numerical results of Chernykh et al. (1998). 


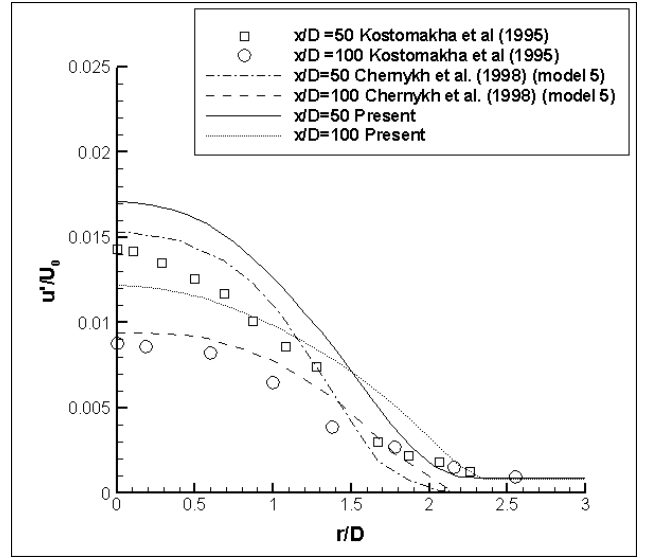

a)

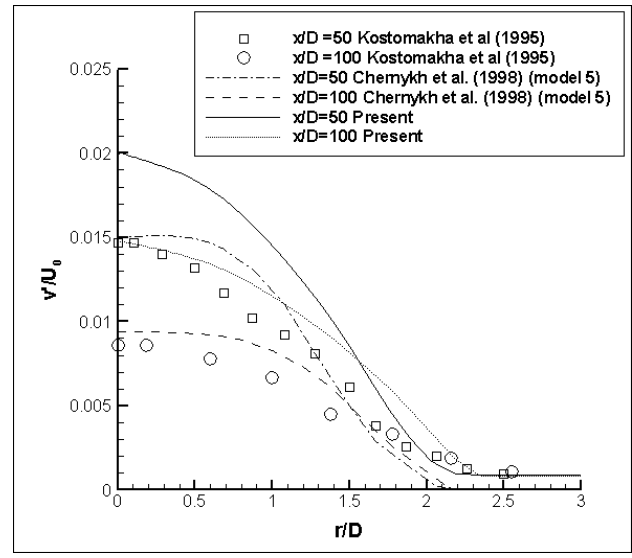

b)

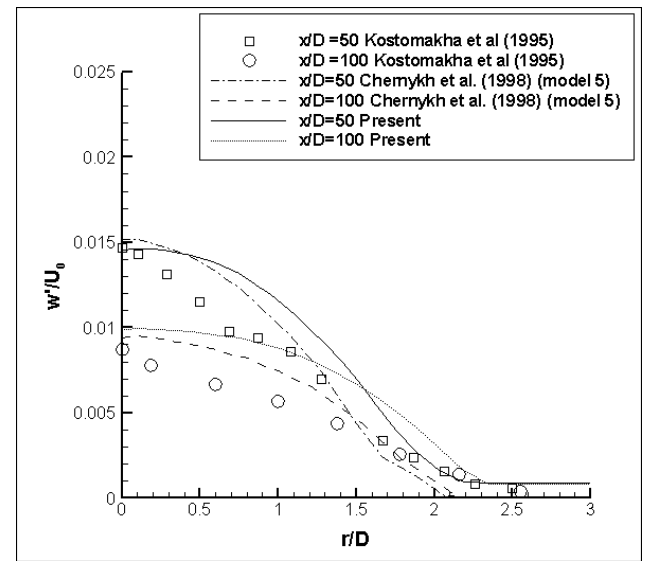

c)

Figure 3. Comparison of the calculated dimensionless profiles of the fluctuation intensities of the velocity components with the experimental data of Kostomakha et al. (1995) and the numerical results of Chernykh et al. (1998).

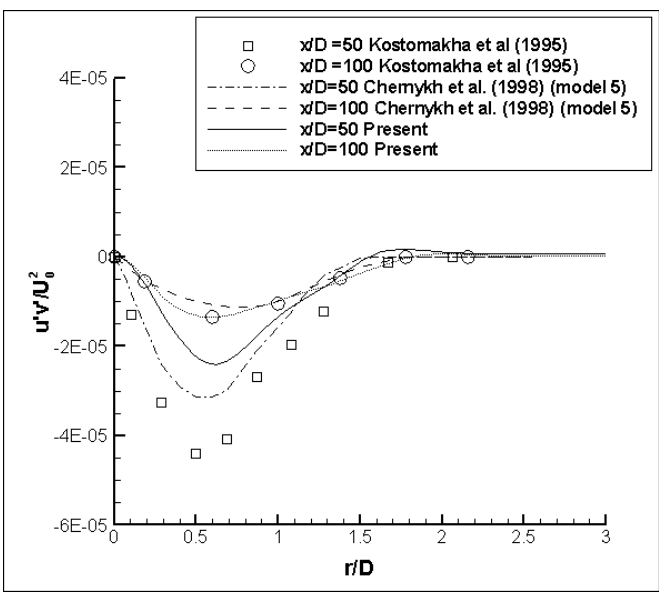

a)

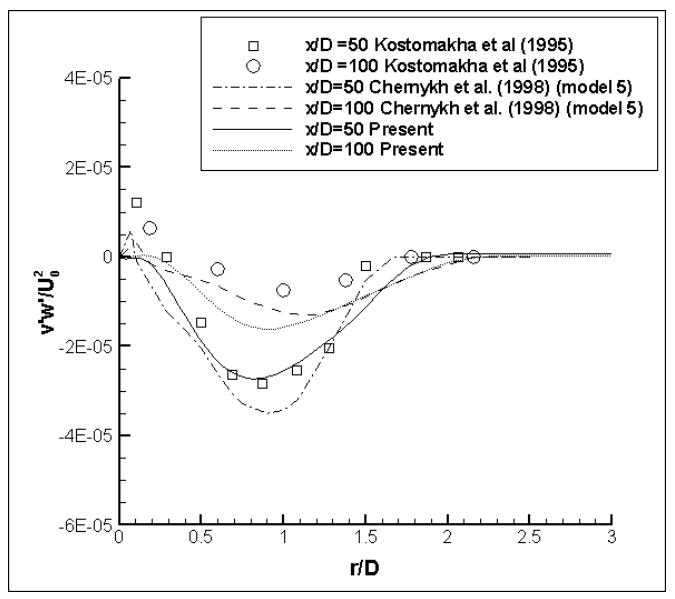

b)

Figure 4. Comparison of the calculated dimensionless profiles of the tangential Reynolds stresses a) uv and b) vw with the experimental data of Kostomakha et al. (1995) and the numerical results of Chernykh et al. (1998). 
To evaluate the ability of the used second order closure to simulate the evolution of the momentumless swirling wake, the decay of the axial and tangential velocity scales was computed and is shown in Figure 5. Comparisons of the results obtained from the experimental study of Kostomakha et al. (1995) and two different numerical solutions (corresponding to models 2 and 5; refer to section II) of Chernykh et al. (1998), as well as with other experimental results (Faure, 1995 and Schetz and Jakubowski, 1975) corresponding to axisymmetric momentumless wakes with different initial conditions, as well as Re and S numbers, are also shown in Figure 5.

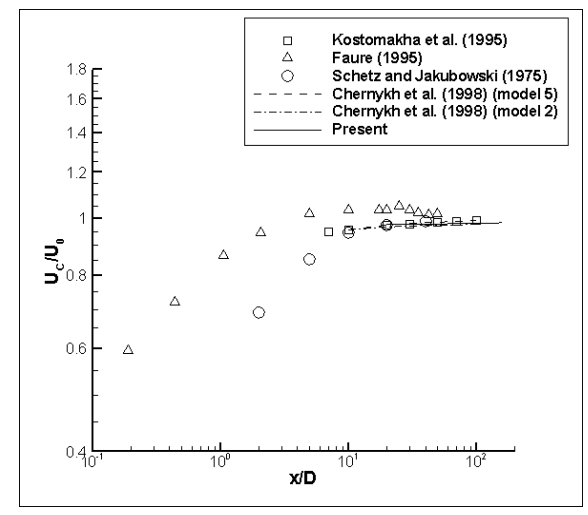

a)

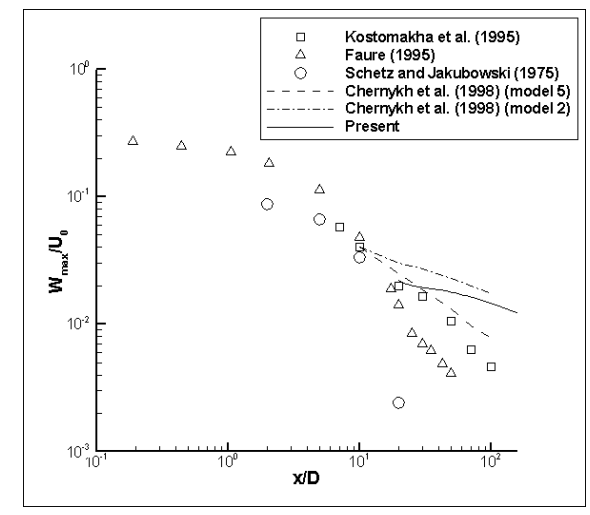

b)

Figure 5. Evolution of wake velocity scales a) centerline velocity and b) maximum tangential velocity, corresponding to axisymmetric momentumless wakes

From Figure 5a it can be observed that within 10D regardless of the differences in the initial boundary conditions corresponding to each of the flows represented, the centerline velocity becomes within $3 \%$ of the free-stream velocity. The small-defect assumption that is conventionally made in classical similarity theory of wakes is met quite early by this canonical flow. The agreement of the numerical predictions among themselves and with the array of experimental results presented in Figure 5a is rather good. On the other hand the decay of the tangential velocity takes place at a comparatively much slower pace than that observed for the streamwise velocity scale. Furthermore the predictions corresponding to the numerical simulations of the model 2 of Chernykh et al. (1998) and the present simulations seem to have a similar slope, however they truly miss the experimental trend as well as the results obtained by the model 5 of Chernykh et al. The latter is in good agreement with the experimental data.

The evolution corresponding to the intensity and length scale of uu is plotted in Figure 6, where the evolution of the maxima corresponding to the streamwise velocity fluctuations is presented. As the maximum value of uu occurs at the centerline, the corresponding length scale, $\mathrm{r}_{1 / 2}$, is taken as the radial distance where the value of the axial turbulence intensity falls to half of its maximum. The $u_{\text {max }}$ takes place at the centerline up to $x=1 D$. From approximately $\mathrm{x}=10 \mathrm{D}$ the decay of the streamwise velocity fluctuations seem to take place at a faster pace emphasized by the slope shown by the experimental data, as well as the numerical predictions. The evolution of the turbulence length scale seems to be well predicted by any of the simulations.

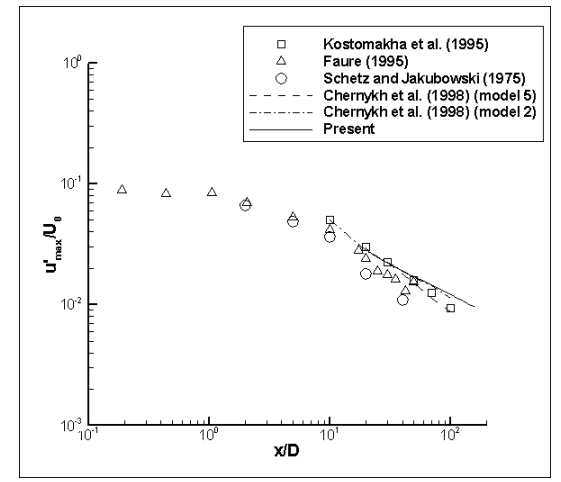

a)

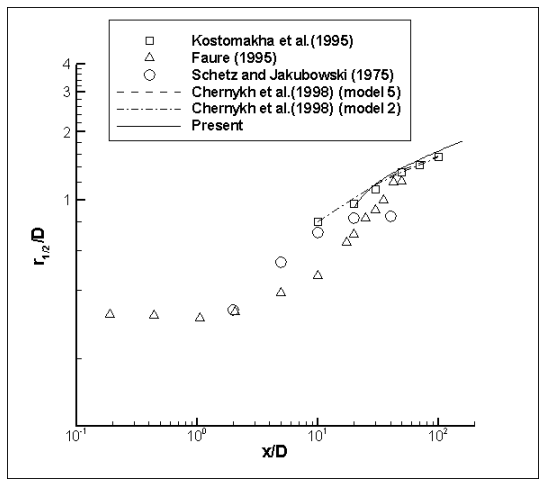

b)

Figure 6. Evolution of the intensity scale of uu and corresponding turbulent length scale for axisymmetric momentumless wakes 
The results of Hyun and Patel (1991) corresponding to a detailed experimental study of an axisymmetric body self-propelled by a three-blade propeller were compared with the experimental data of Sirviente and Patel (2000). The latter data base correspond to the same body as that used by Hyun and Patel but self-propelled with a swirling jet instead for the same Re and S. Both cases were simulated with the present numerical approach and comparisons are shown for the evolution of the centerline velocity and the maximum tangential velocity in Figure 7. Comparisons of velocity and turbulence profiles for two different locations $x / D_{p}=1.87$ and 7.12 , where $D_{p}$ represents the diameter of the propeller, are shown in Figure 8. Both figures 7 and 8 include some of the numerical predictions of the Hyun and Patel data performed by Stern et al. (1991) with a $\kappa-\varepsilon$ model. Figure 7 indicates that the Reynolds stress closure performs better in the prediction of the evolution of the decay of the maximum tangential velocity than of the centerline velocity, which seems to be better predicted by the isotropic turbulence closure.
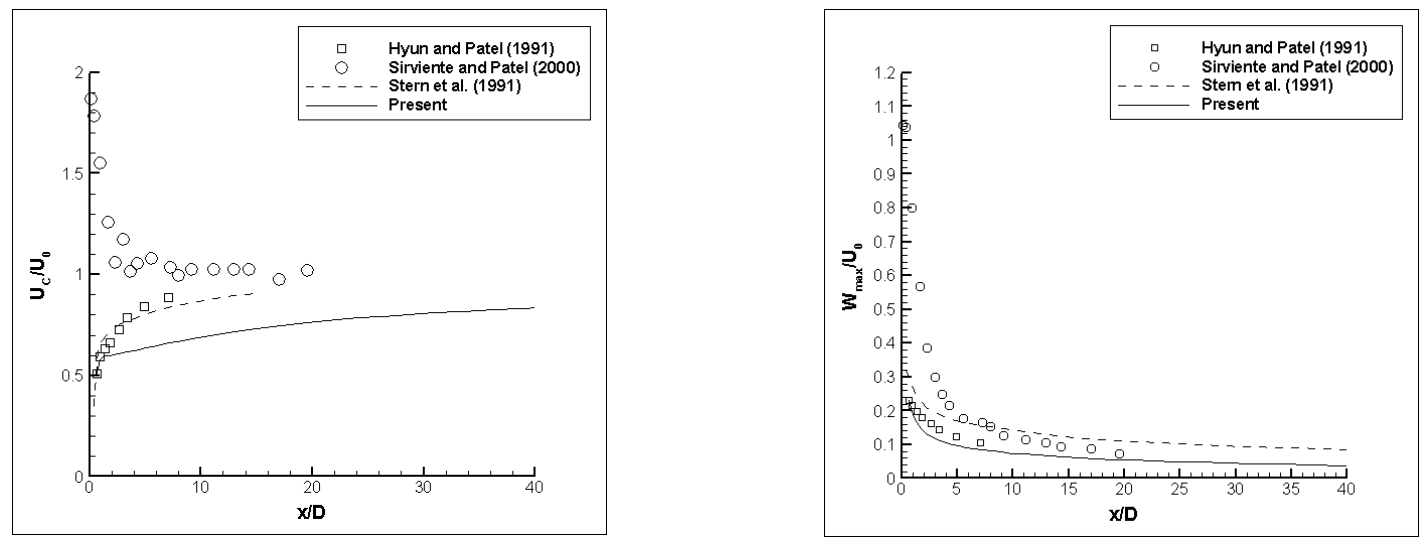

Figure 7. Comparison of the evolution of the wake velocity scales corresponding to the axisymmetric momentumless wake data of Hyun and Patel (1991) and Sirviente and Patel (2000) with the present simulation

The results presented in Figure 8 show in general a much better agreement of the present simulation than that corresponding to the $\kappa-\varepsilon$ model for both velocity and turbulence predictions. It should be noted that the radial velocity fluctuations are larger than the streamwise and tangential velocity fluctuations respectively by $\mathrm{x} / \mathrm{D}_{\mathrm{p}}=1.87$, while the trend has changed ( $\left.u^{\prime}>v^{\prime}>w^{\prime}\right)$ by $7.12 D_{p}$.

\section{B. Wake of an Axisymmetric Body}

The wake of an axisymmetric body is characterized by an axial momentum deficit which corresponds to the drag of the body. The data of Hyun and Patel (1991) also includes measurements of the three velocity components and the six Reynolds stresses of the wake of the axisymmetric body without the propeller, for the same Reynolds number as that employed for the zero-momentum wake measurements. The ability of the second order closure to properly predict the development of the wake of this bare hull was tested as well.

The comparisons of the velocity and turbulence profiles corresponding to $\mathrm{x} / \mathrm{D}_{\mathrm{p}}=1.87$ and 7.12 are presented in Figure 9, along with the corresponding simulations performed by Stern et al. (1991) using a $\kappa-\varepsilon$ model. The results corresponding to the decay of the centerline velocity and of $r_{1 / 2}$ are shown in Figure 10. From Figure 9 it can be observed a significant overprediction of the normal stresses in comparison to the turbulence kinetic energy obtained by the $\kappa-\varepsilon$ model employed by Stern et al. The latter computations were started over the body and extended into the wake. The present simulations on the other hand made use of the experimental data gathered in the near wake $(\mathrm{x} / \mathrm{Dp}=0.3675)$ as inlet boundary condition. The evolution of the centerline velocity seems to be better predicted by the $\kappa-\varepsilon$ model, at least in the near wake.

The results obtained from the present simulation were also compared with those calculated using the comercial software Fluent with a second order closure. The discrepancies obtained for the normal and Reynolds stresses in comparison to the experimental data of Hyun and Patel were comparable to those shown in Figure 9. The impact of the inlet boundary condition used in this computation was not tested. That is assessment of the results obtained with the next available experimental profile was not done. The gradients in the near wake region are important and consequently the experimental errors associated with intrusive hot-wire anemometry are larger than in the far wake. The study of the effect of the inlet boundary condition deserves further investigation. 

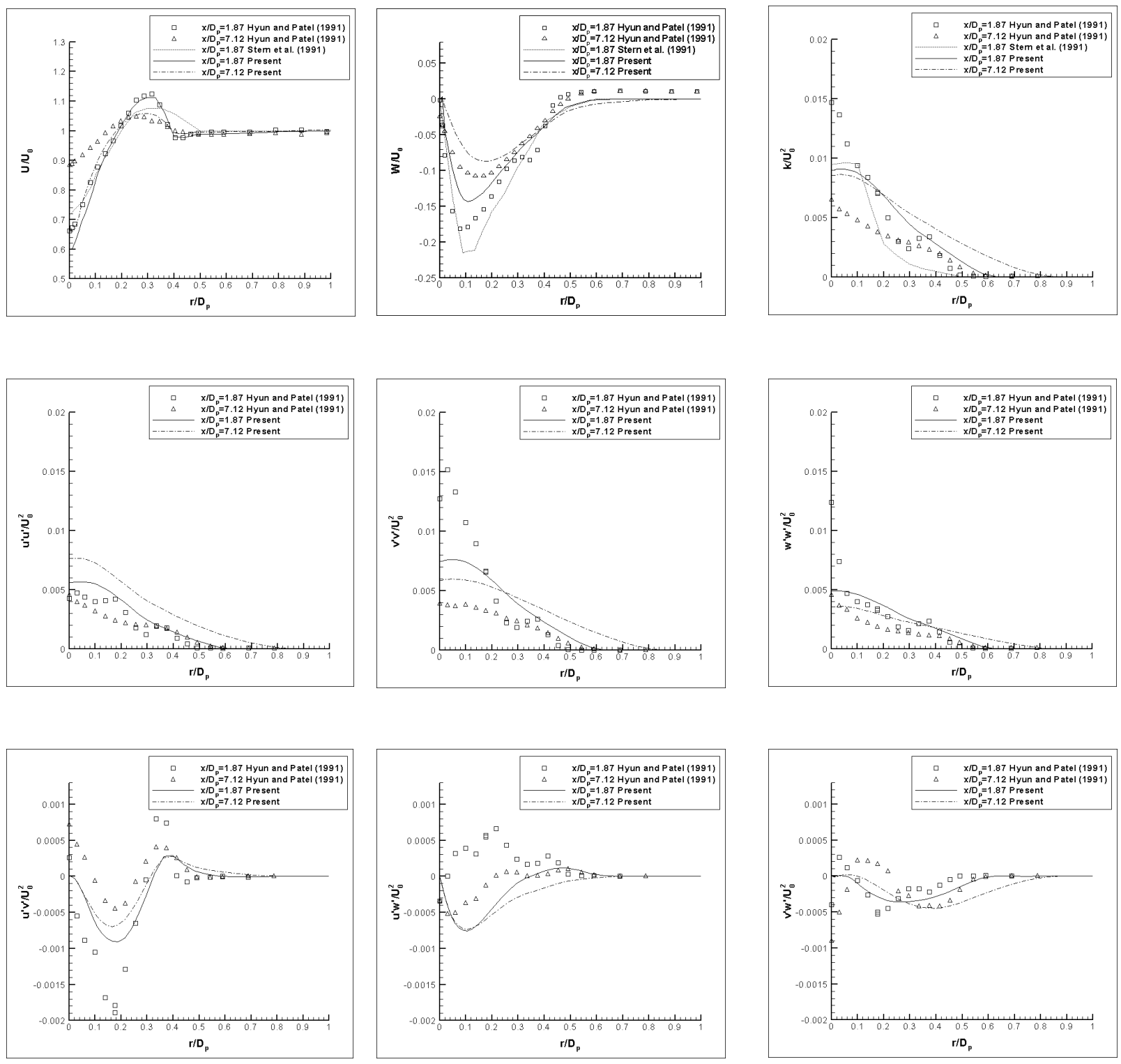

Figure 8. Comparisons of the velocity and turbulence profiles of the momentumless swirling wake for $x / D_{p}=1.87$ and 7.12 with the experimental data of Hyun and Patel (1991) and the numerical model of Stern et al. (1991)

\section{Isolated Circular Jet}

Simulations corresponding to an axisymmetric jet were also done. The experimental data of Sami et al. (1967) at $x / D=3$ was used as inlet boundary condition. The Reynolds number in this case was $R e=2.2 \times 10^{5}$. The purpose of this numerical experiment was to compare the performance of the second order turbulence closure for this canonical flow, which can be viewed in a simplistic manner as representative of the means of propulsion of a self-propelled body. Nonetheless the conditions of the experiment of Sami et al. did not correspond to the axial momentum constraints to match the Hyun and Patel study. Nonetheless the simultions were takled as representative of the general trends shown by the turbulence closure for this type of free shear layers. 

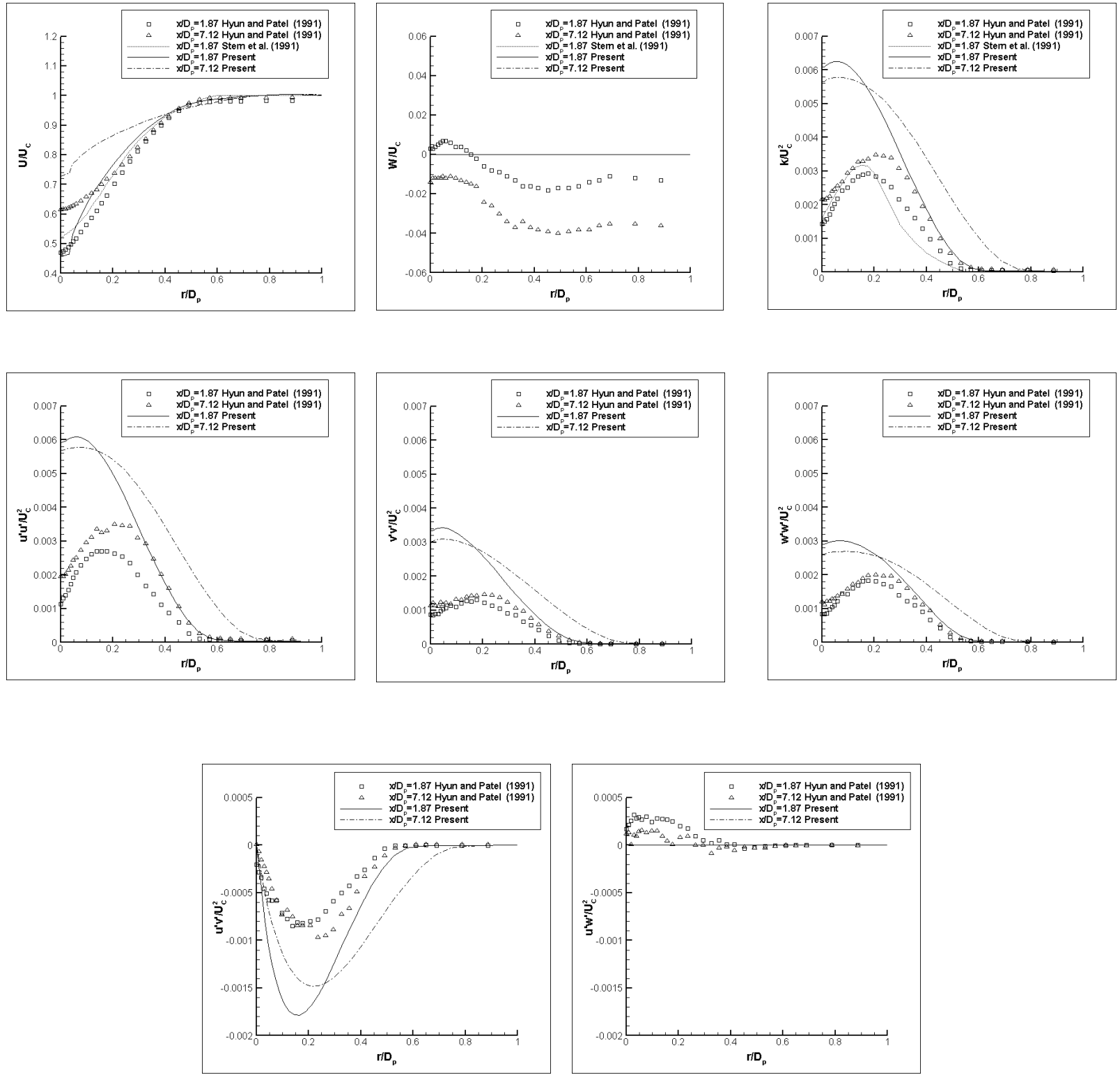

Figure 9. Comparisons of the velocity and turbulence profiles of the wake of an axisymmetric body for $\mathrm{x} / \mathrm{D}_{\mathrm{p}}=1.87$ and 7.12 with the experimental data of Hyun and Patel (1991) and the numerical model of Stern et al.

The results corresponding to the decay of the centerline velocity and of $r_{b}$, defined as the radial distance where the value of mean axial velocity is half of the difference between centerline and free stream velocity are shown in Figure 11. The trends shown by the centerline velocity are clearly consistent with other experimental data. On the other hand the numerical results corresponding to $r_{b}$ initially agree with the experimental data of Sami et al. but there is not enough experimental data in the wake to perform a detail validation. The numerical data corresponding to rather weak swirling jets, also shown in the figure, indicates a different evolution for the non-swirling jet. The comparisons of the velocity and turbulence profiles corresponding to various steamwise locations are presented in Figure 12, along with the experimental data of Sami et al. (1967) and Hussein et al. (1994). Figure 12 indicates that the mean velocity becomes self-similar quite early by $\mathrm{x} / \mathrm{D}=40$, while the turbulence requires a longer streamwise 
distance to achieve self-similarity, at least 75D. The predictions of the normal and turbulent stresses are quite good in comparison to the experimental data.
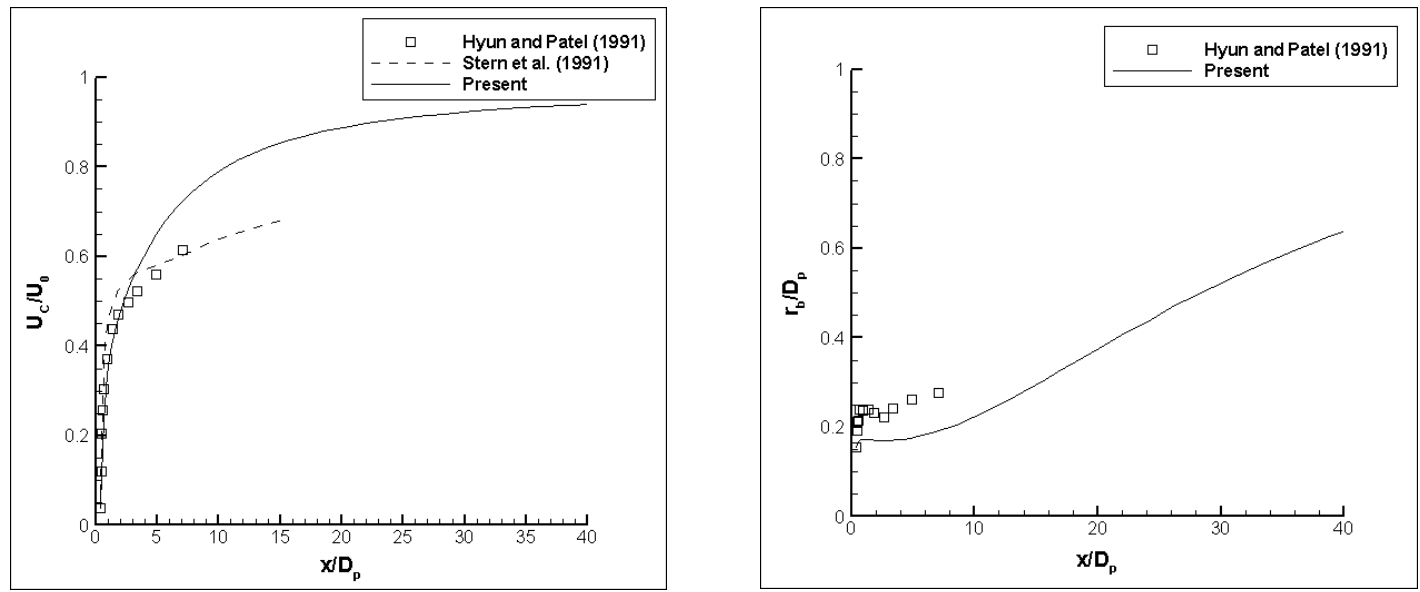

Figure 10. Comparison of the decay of the centerline velocity and the $r_{b}$ length scale corresponding to the wake of the axisymmetric body of Hyun and Patel (1991)
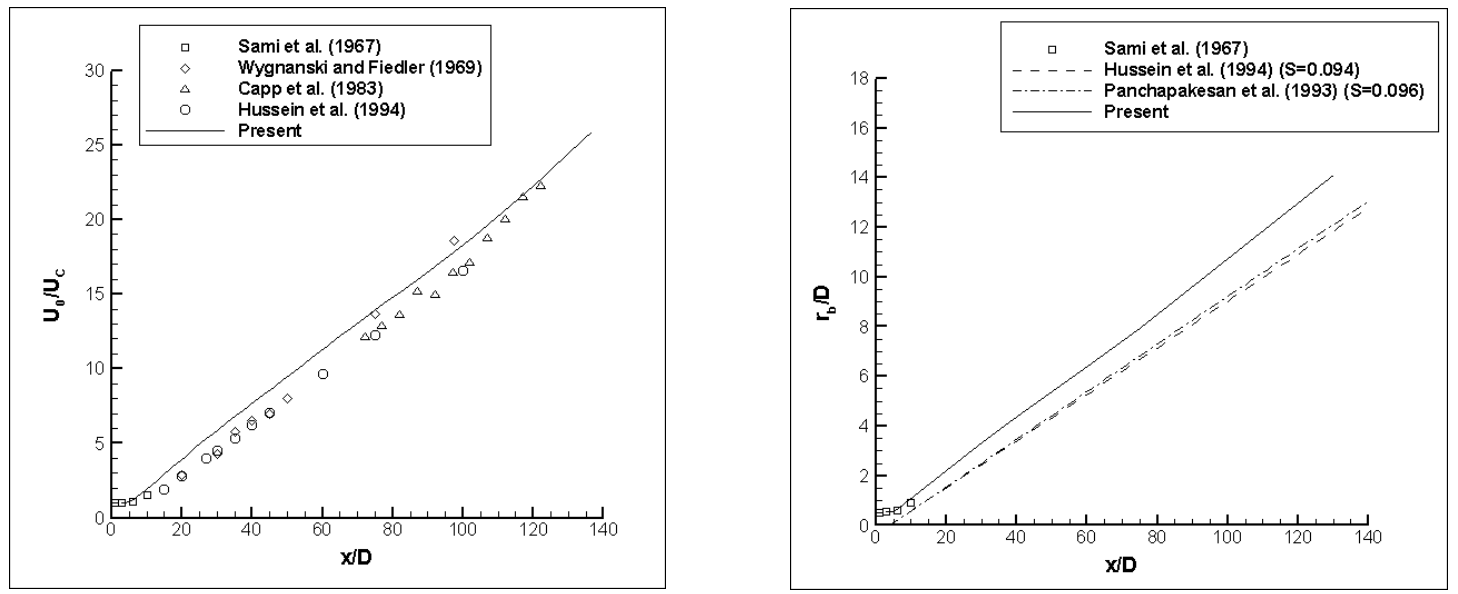

Figure 11. Comparison of the decay of the centerline velocity and the $\mathrm{r}_{\mathrm{b}}$ length scale corresponding to an axisymmetric jet

\section{Summary and Conclusions}

A Reynolds-averaged Navier-Stokes solver in conjuction with a second order Reynolds stress closure was used to calculate the turbulent flow of a swirling axisymmetric momentumless wake. The computed results were compared with various experimental and numerical data bases. The same numerical model was also use to numerically simulate the so called component flows of the zero-momentum wake, that is the wake of the bare body and the jet like type of flow generated by the means of propulsion.

The following conclusions can be drawn from the present study: 

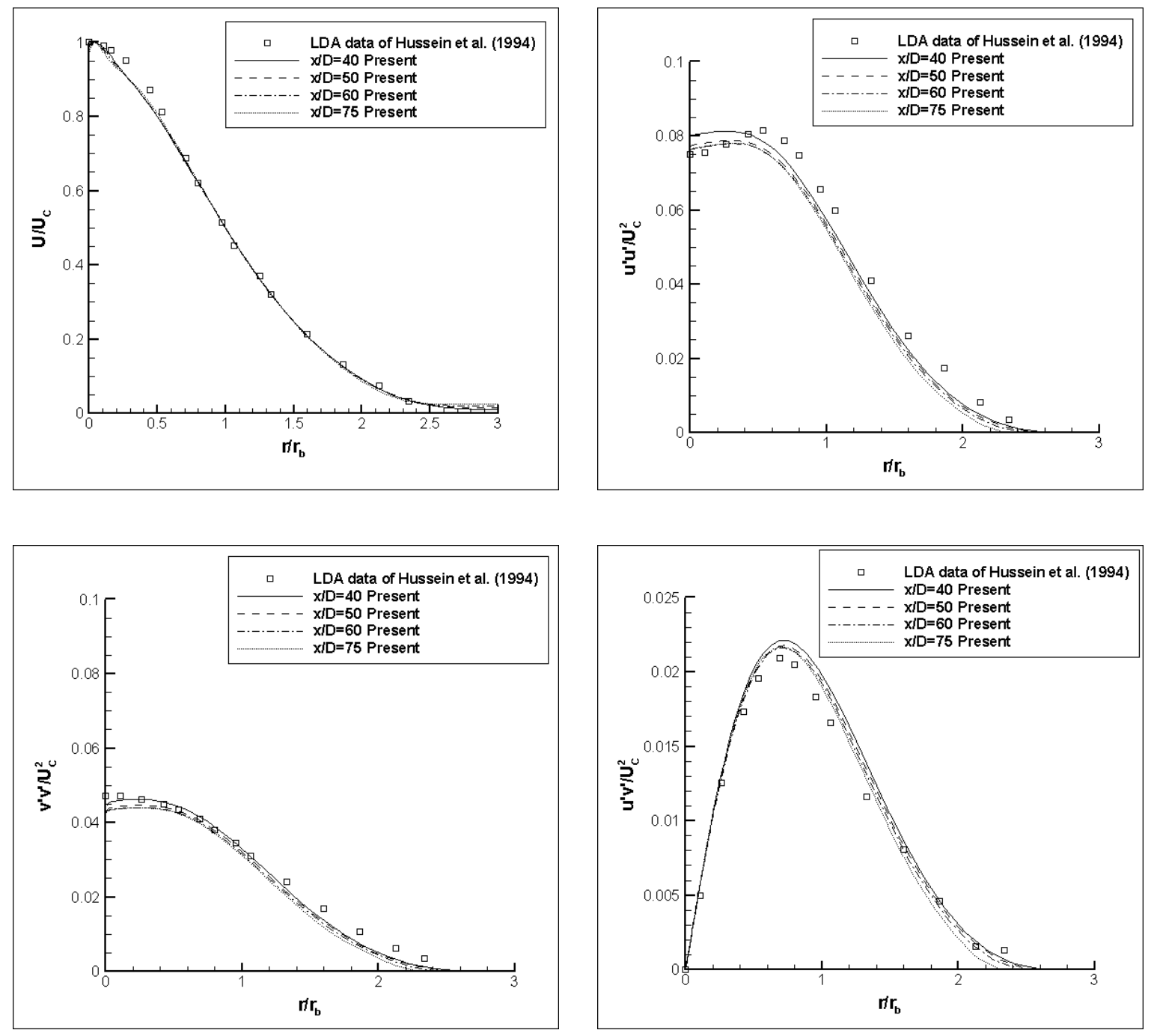

Figure 9. Comparisons of the velocity and turbulence profiles of the axisymmetric jet for various locations with the experimental data of Hussein et al. (1994)

1. The present computations reproduce, with remarkable accuracy, the evolution of the mean and turbulent characteristics corresponding to the axisymmetric jet flow studied as one of the component flows of the axisymmetric momentumless wake.

2. These numerical experiments showed that the simulations corresponding to the axisymmetric momentumless wake and wake of the bare body tend to overpredict the normal and Reynolds stresses in comparison with experimental data. The trends found in both computations were remarkably similar. This seems to indicate that the inability of the second order closure to accurately capture the correct evolution of the zero-momentum wake is linked to its performance in the simulation of the bare body wake.

3. One of the models employed by Chernykh et al. (1998) for the simulation of a swirling axisymmetric momentumless wake was able to produce better predictions than the present model for the normal stresses of the swirling axisymmetric momentumless wake. 


\section{References}

1. Cimbala, J.M., and Park, W.J., (1990), "Experimental Investigation of the Turbulent Structure in a Two Dimensional Momentumless Wake," Journal of Fluid Mechanics, Vol. 213, pp. 479-509

2. Park, W.J., and Cimbala, J.M., (1991), "Effect of jet Injection Geometry on Two-Dimensional Momentumless Wakes," Journal of Fluid Mechanics, Vol. 224, pp. 29-47.

3. Ridjanovic, M., (1963), "Wake with Zero Change of Momentum Flux," Ph.D. Dissertation, Dept. of Mechanical Engineering, University of Iowa, Iowa City, IA.

4. Wang, H., (1965), "Flow Behind a Point Source of Turbulence," Ph.D. Disseration, Department of Mechanical Engineering, University of Iowa, Iowa City, IA.

5. Naudascher, E., (1965), "Flow in the Wake of Self-Propelled Bodies and Related Sources of Turbulence," Journal of Fluid Mechanics, Vol. 22, pp. 625-656.

6. Higuchi, H., and Kubota, T., (1990), "Axisymmetric Wakes Behind a Slender Body Including ZeroMomentum Configurations," Physics of Fluids A-Fluid Dynamics, Vol. 2, pp. 1615-1623.

7. Schetz, J.A., and Jakubowski, A.K., (1975), "Experimental Studies of the Turbulent Wake behind SelfPropelled Slender Bodies," AIAA Journal, Vol. 13(12), pp. 1568-1575.

8. Ferry, M., and Piquet, J., (1987), "Sillage Visqueux Lointain d'un Corps Sous-Marin Autopropulse," SIREHNA, Rapport d'Ettude 86/14R, Nantes, France.

9. Faure, T.M., (1995), "Etude experimentale du sillage turbulent d'un corps a symetrie de revolution autopropulse par helice." Ph.D. Thesis, No 95-01, Ecole Centrale de Lyon.

10. Hyun, B.S., and Patel, V.C., (1991), "Measurements in the Flow around a Marine Propeller at the Stern of an Axisymmetric Body," Experiments in Fluids, Vol. 11, pp. 33-44.

11. Sirviente, A.I. and Patel, V.C. (2001), "Turbulence Structure of the Wake of a Self- Propelled Body with and without Swirl," AIAA Journal, Vol. 39(12), pp. 2411-2414.

12. Sirviente, A.I. and Patel, V.C. (2000), "Wake of a Self-Propelled Body. Part I: Momentumless Wake," AIAA Journal, Vol. 38(4), pp. 613-619; Part II: Momentumless Wake with Swirl," AIAA Journal, Vol. 38, pp. 620-627.

13. Kostomakha, V.A., and Lesnova, N.V., (1995), "Turbulent Swirling Wake behind a Sphere with Complete or Partial Drag Compensation," Journal of Applied Mechanics and Technical Physics, Vol. 36(2), pp. 226-233.

14. Schooley, A.H., and Stewart, R.W., (1963), "Experiments with a Self-Propelled Body Submerged in a Fluid with Vertical Density Gradient," J Journal of Fluid Mechanics, Vol. 15, pp. 83-96.

15. Gilreath, H.E., and Brandt, A., (1985), "Experiments on the Generation of Internal Waves in a Strafied Fluid," AIAA Journal, Vol.23(5), pp. 693-700.

16. Chernykh, G.G., Ilyushin, B.B., and Voropayeva, O.F. (2003), "Anisotropy Decay of Turbulence in a Far Momentumless Wake in a Linearly Stratified Medium," Russ. J. Numer. Anal. Math. Modeling, Vol. 18, pp. 105116.

17. Tennekes, H., and Lumley, J. L., (1972), A First Course in Turbulence, MIT Press, Cambridge, MA.

18. Finson, M.L., (1975), "Similarity Behavior of Momentumless Turbulent Wakes," Journal of Fluid Mechanics, Vol. 71, pp. 465-479.

19. Hassid, S., (1980), "Similarity and Decay Law of Momentumless Wakes," Physics of Fluids, Vol. 23, No. 2, pp. 404-405.

20. Ferry, M., and Piquet, J., (1987), "Sillage Visqueux Lointain d'un Corps Sous-Marin Autopropulse," SIREHNA, Rapport d'Ettude 86/14R, Nantes, France.

21. Sirviente, A. I., (1996), "Wake of an AxisymmetricBody Propelled by a Jet with and without Swirl," Ph.D. Dissertation, Dept. of Mechanical Engineering, Univ. of Iowa, Iowa City, IA.

22. Chernykh, G.G., Demenkov, A.G., and Kostomakha, V.A., (1998), "Numerical Model of a Swirling Momentumless Turbulent Wake," Russ. J. Numer. Anal. Math. Modeling, Vol. 13, pp. 279-288.

23. Chernykh, G.G., and Voropayeva, O.F., (1999), "Numerical Modeling of Momentumless Turbulent Wake Dynamics in a Linearly Stratified Medium," Computers \& Fluids, Vol. 28, pp. 281-306.

24. Stern, F., Toda, Y., and Kim, H.T., (1991), "Computation of Viscous Flow around Propeller-Body Configurations: Iowa Axisymmetric Body," Journal of Ship Research, Vol. 35, pp. 151-161.

25. Sotiropoulos, F., and Abdallah, S., (1991), "The discrete continuity equation in primitive variable solutions of incompressible flow," J. of Computational Physics, Vol. 95, pp. 212-227.

26. Sotiropoulos, F. and Patel, V.C., (1992), "Flow in Curved Ducts of Varying Cross-Section," IIHR Report no. 358 . 
27. Sinha, S.K., Sotiropouslos, F. and Odgaard, A.J., (1998), "Three-dimensional Numerical Model for Flows Through Natural Rivers," Journal of Hydraulic Engineering, Vol. 124, pp. 13-24.

28. Kim, K., Sirviente, A.I. and Beck, R.F., (2005), "The complementary RANS equations for the Simulation of Viscous flows," International Journal for Numerical Methods in Fluids (in press).

29. Hanjalic, K., and Launder, B.E., (1976), "Contribution toward a Reynolds-Stress Closure for LowReynolds-Number Turbulence,” Journal of Fluid Mechanics, Vol. 74, pp. 593-610.

30. Launder, B.E., and Shima, N., (1989), "Second-Moment Closure for the Near-Wall Sublayer. Development and Application,” AIAA Journal, Vol. 27, pp. 1319-1325.

31. Sami, S., Carmody, T., and Rouse, H., (1967), "Jet Diffusion in the Region of Flow Establishment," Journal of Fluid Mechanics, Vol. 27, pp. 231-252.

32. Launder, B.E., and Morse, A., (1979), "Numerical Prediction of Axisymmetric Free Shear Flows with a Second-Order Reynolds Stress Closure,” In Turbulent Shear Flows, 1, Springer, Berlin, pp. $279-294$. 